\title{
Effects of Sintering Temperature on Thermoelectric Device of La-Doped Strontium Titanate in the Combination of Combustion Synthesis and Spark Plasma Sintering
}

\author{
Asami Kikuchi ${ }^{1, *}$, Lihua Zhang ${ }^{2}$, Noriyuki Okinaka ${ }^{3}$, Tsuyoshi Tosho ${ }^{3}$ and Tomohiro Akiyama ${ }^{3}$ \\ ${ }^{1}$ Graduate School of Engineering, Hokkaido University, Sapporo 060-8628, Japan \\ ${ }^{2}$ University of Science and Technology Beijing, Beijing, 100083, P.R. China \\ ${ }^{3}$ Center for Advanced Research of Energy Conversion Materials, Hokkaido University, Sapporo 060-8628, Japan
}

Thermoelectric properties of La-doped $\mathrm{SrTiO}_{3}$ were investigated in order to determine the optimum sintering temperature for its fabrication by using a combination of combustion synthesis and spark plasma sintering. Combustion-synthesized samples $\left(\mathrm{Sr}_{1-x} \mathrm{La}_{x} \mathrm{TiO}_{3}\right.$, $x=0.08$ ) were subjected to spark plasma sintering at temperatures from 1513 to $1663 \mathrm{~K}$. The average grain size of sintered $\mathrm{Sr}_{0.92} \mathrm{La}_{0.08} \mathrm{TiO}_{3}$ enlarged as sintering temperature rose up. The maximum average grain size was $23.5 \mu \mathrm{m}$ for a sintering temperature of $1663 \mathrm{~K}$. The thermoelectric properties of sintered $\mathrm{Sr}_{0.92} \mathrm{La}_{0.08} \mathrm{TiO}_{3}$ were measured from room temperature to $1073 \mathrm{~K}$. The optimum sintering temperature in the experimental sintering temperature range was $1633 \mathrm{~K}$. Among the samples, the $\mathrm{Sr}_{0.92} \mathrm{La}_{0.08} \mathrm{TiO}_{3}$ sample sintered at $1633 \mathrm{~K}$ showed the maximum power factor of $1.51 \times 10^{-3} \mathrm{Wm}^{-1} \mathrm{~K}^{-1}$ at $375 \mathrm{~K}$. Further, we investigated the effects of pressing direction during sintering on the thermoelectric properties of combustion-synthesized $\mathrm{Sr}_{0.92} \mathrm{La}_{0.08} \mathrm{TiO}_{3}$. The combustion-synthesized samples were sintered well along the pressing direction during sintering; therefore, the electric conductivity measured along the pressing direction during sintering was more than twice that measured along the direction perpendicular to the pressing direction during sintering. Thus, we concluded that pressing direction during sintering affected the electric property of $\mathrm{Sr}_{0.92} \mathrm{La}_{0.08} \mathrm{TiO}_{3}$. [doi:10.2320/matertrans.M2009225]

(Received June 30, 2009; Accepted September 4, 2009; Published October 15, 2009)

Keywords: thermoelectric materials, perovskite oxide, combustion synthesis, spark plasma sintering

\section{Introduction}

The last decade has seen a big surge in research on thermoelectric oxide materials, with primary focus on improving the thermoelectric properties: electric conductivity, $\sigma\left[\mathrm{Scm}^{-1}\right]$, Seebeck coefficient, $\alpha\left[\mu \mathrm{VK}^{-1}\right]$, and thermal conductivity, $\kappa\left[\mathrm{Wm}^{-1} \mathrm{~K}^{-1}\right]$. The term $\alpha^{2} \sigma$ is called power factor $\left[\mathrm{Wm}^{-1} \mathrm{~K}^{-2}\right] . \mathrm{SrTiO}_{3},{ }^{1-4)}$ a typical transition-metal perovskite oxide, is one of the most important ceramics. ${ }^{1)}$ Okuda et $a l .{ }^{5)}$ have studied the thermoelectric properties of single-crystal $\mathrm{Sr}_{1-x} \mathrm{La}_{x} \mathrm{TiO}_{3}(0 \leq x \leq 0.1)$ and found that it has a large power factor of $3.6 \times 10^{-3} \mathrm{Wm}^{-1} \mathrm{~K}^{-2}$ at room temperature, which is comparable to that of practical bismuth telluride alloys. In order to improve the thermoelectric performance of thermoelectric materials, other elements have been partially substituted for $\mathrm{Sr}$ and $\mathrm{Ti}$ site in the reported study. ${ }^{6-9)}$

Polycrystalline $\mathrm{SrTiO}_{3}$ is usually synthesized by the conventional solid-state reaction (SSR) method and sol-gel method, which have already been widely used in the production of many materials. However, conventional methods involve many processes and are therefore time and energy consuming. Zhang et al. ${ }^{1,10}$ have proposed a combination of combustion synthesis $(\mathrm{CS})^{11)}$ and spark plasma sintering (SPS) ${ }^{12-17)}$ for the synthesis of thermoelectric materials. As compared to conventional techniques such as hot-pressed sintering, SPS is highly suitable for the fabrication of thermoelectric materials. ${ }^{18,19)}$ Sintering conditions, particularly the sintering temperature, seriously affect the microstructures and properties of materials. ${ }^{18,20,21)}$ However, the effects of sintering temperature on the thermoelectric properties of thermoelectric materials have not been

*Graduate Student, Hokkaido University investigated so far. Furthermore, thermoelectric materials conduct heat and electricity along the same direction; therefore, it is essential to consider the pressing direction during sintering, particularly the products fabricated by SPS. Therefore, in this study, we investigated the effects of sintering temperature on the thermoelectric properties and microstructures of polycrystalline $\mathrm{Sr}_{0.92} \mathrm{La}_{0.08} \mathrm{TiO}_{3}$ and the effects of pressing direction during sintering on its thermoelectric properties and microstructures.

\section{Experimental Procedure}

Polycrystalline samples of La-doped $\mathrm{SrTiO}_{3}$ (SLTO) were prepared from $\mathrm{SrCO}_{3}(99.9 \%$ purity, Kanto Chemical, Tokyo, Japan), $\mathrm{TiO}_{2}$ (99.9\% purity, Kanto Chemical, Sakado, Japan), Ti (99.9\% purity, Kojundo Chemical, Sakado, Japan), $\mathrm{NaClO}_{4}$ (98.0\% purity, Sigma-Aldrich, St. Louis, USA), and $\mathrm{La}_{2} \mathrm{O}_{3}$ (99.9\% purity, Kojundo Chemical, Sakado, Japan). ${ }^{1)}$ After combustion-synthesis, the obtained products were pulverized into powders by using a zirconia mortar and pestle and ground in a planetary ball mill (Pulverisette 6, Fritsch, Idor-Oberstein, Germany) operated at $350 \mathrm{rpm}$ for $40 \mathrm{~min}$ in air. The particle size of the obtained powders was within $5 \mu \mathrm{m}$.

The obtained powders were sintered by SPS (SPS-511S, Sumitomo Coal Mining, Tokyo, Japan). The combustionsynthesized and pulverized powders were filled in a cylindrical graphite die (inner diameter $=15 \mathrm{~mm}$ ) and pressed by using a graphite punch at $37.45 \mathrm{MPa}$. A graphite foil was placed inside the graphite die before filling it with the powders so that the sintered powders can be easily extracted. In addition, the die was covered with graphite felt to minimize heat loss by thermal radiation. Sintering was carried out at a heating rate of $30 \mathrm{Kmin}^{-1}$ and a holding time 

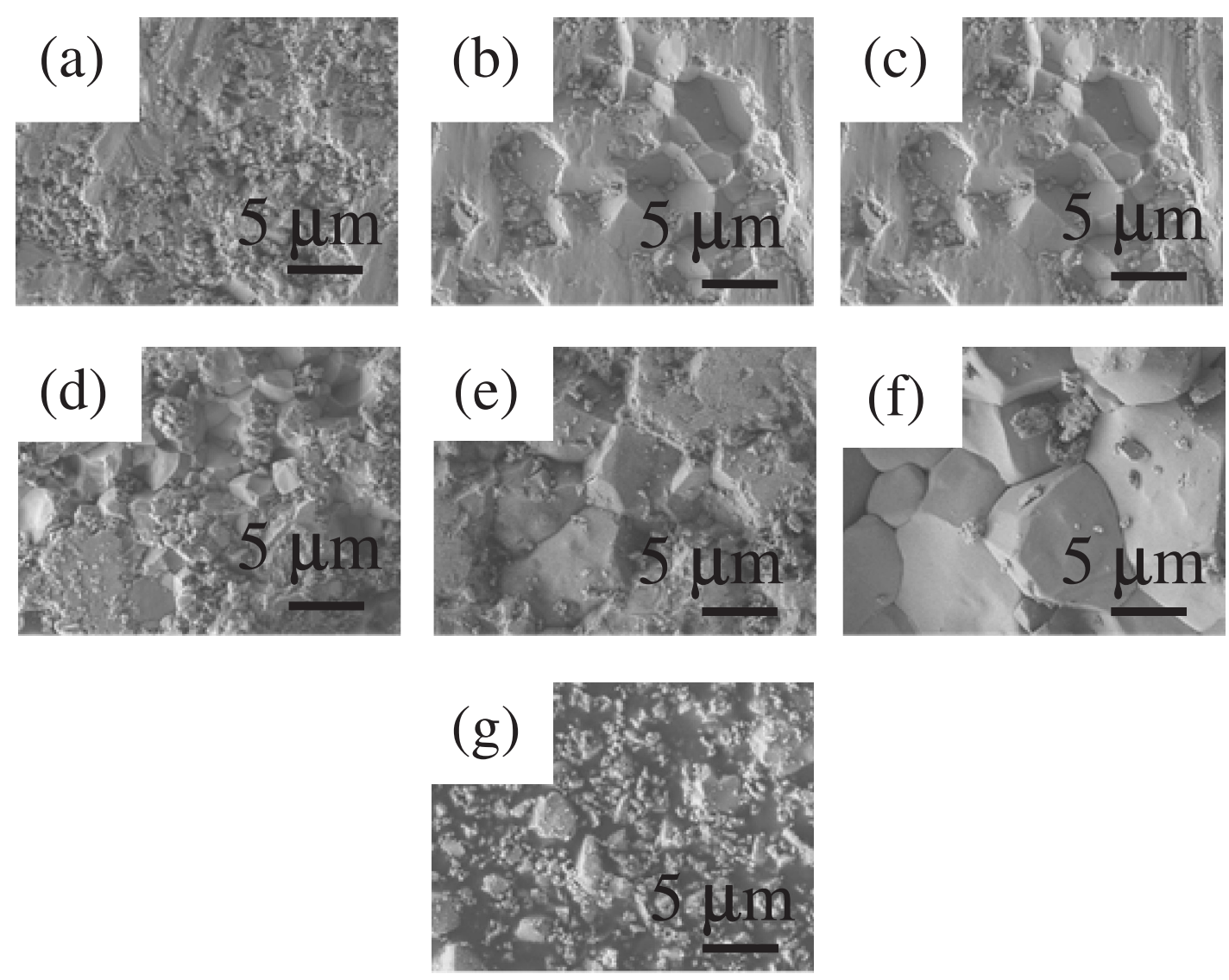

Fig. 1 SEM images of a cross section of SLTO sintered at (a) $1513 \mathrm{~K}$, (b) $1543 \mathrm{~K}$, (c) $1573 \mathrm{~K}$, (d) $1603 \mathrm{~K}$, (e) $1633 \mathrm{~K}$, (f) $1663 \mathrm{~K}$, and (g) is an SEM image of the powders before sintering.

of $15 \mathrm{~min}$, which is the same as the holding time in vacuum. During sintering, the mechanical pressure was maintained at $34 \mathrm{MPa}$ by using plungers. The temperature was measured by means of an optical pyrometer attached to the surface of the graphite die. In this study, sintering was carried out at different holding temperatures.

The oxygen defect content $\delta$ in the sintered samples was determined by measuring the weight of the samples before and after heating in the furnace at $1373 \mathrm{~K}$ for $12 \mathrm{~h}$ in air. Heating was repeated twice. $\delta$ was calculated assuming that the samples were oxidized to $\mathrm{Sr}_{0.92} \mathrm{La}_{0.08} \mathrm{TiO}_{3.00}$ in furnace.

The phase composition and morphology of the products were analyzed by using an X-ray diffractometer (Miniflex, Rigaku, Tokyo, Japan) and a scanning electron microscope (SEM) (JSM-7000F, JEOL, Tokyo, Japan). $\sigma$ and $\alpha$ were simultaneously measured by using a Seebeck coefficient/ electric resistance measuring system (ZEM-3, ULVACRIKO, Yokohama, Japan) from room temperature to $1073 \mathrm{~K}$ in vacuum.

\section{Results and Discussion}

\subsection{Reaction analyses and crystal structure of combus- tion-synthesized (CSed) and spark-plasma-sintered (SPSed) SLTO}

In this paper, CSed and SPSed SLTO indicates the combustion-synthesized and spark-plasma-sintered SLTO. The equation of the CS reaction is given as follows: ${ }^{1,10)}$

$$
\begin{aligned}
& (1-x) \mathrm{SrCO}_{3}+(1-a) \mathrm{Ti}+a \mathrm{TiO}_{2} \\
& +(x / 2) \mathrm{La}_{2} \mathrm{O}_{3}+(4-4 a-x) / 8 \mathrm{NaClO}_{4} \\
& \quad \rightarrow \mathrm{Sr}_{1-x} \mathrm{La}_{x} \mathrm{TiO}_{3}+(1-x) \mathrm{CO}_{2}+(4-4 a-x) / 8 \mathrm{NaCl}
\end{aligned}
$$

In eq. (1), $x$ denotes the La-doping content and a denotes $\mathrm{TiO}_{2}$ content. In our study, $x$ and $a$ are equal to $0.08^{1)}$ and $0.25,{ }^{11)}$ respectively. Figure 1 shows SEM images of a cross section of SLTO sintered at (a) $1513 \mathrm{~K}$, (b) $1543 \mathrm{~K}$, (c) $1573 \mathrm{~K}$, (d) $1603 \mathrm{~K}$, (e) $1633 \mathrm{~K}$, and (f) $1663 \mathrm{~K}$; Fig. 1 (g) shows an SEM image of the powders before sintering. The CSed samples are porous, and the average particle size of the ground powders before SPS is found to be less than $5 \mu \mathrm{m}$ from the SEM image shown in Fig. 1(g). As shown in Fig. 1, the grain size increases with the sintering temperature. The average grain size of SLTO sintered at 1513, 1543, 1573, 1603,1633 , and $1663 \mathrm{~K}$ is approximately $1.32,4.31,3.61$, $2.32,6.63$, and $23.5 \mu \mathrm{m}$, respectively. After SPS, the color of the samples sintered at temperatures above $1543 \mathrm{~K}$ changes from dark gray to light gray, and their surface shows a metallic luster. On the other hand, the color of the sample sintered at $1513 \mathrm{~K}$ remains unchanged, i.e., dark gray, and there is no metallic luster.

Table 1 lists the lattice parameters of the synthesized powders before SPS calculated from X-ray diffraction (XRD) data and the sintering temperature and bulk density after SPS. The density and relative density of CSed SLTO sintered at different temperatures are also listed in Table 1. The density of the samples sintered at temperatures above $1543 \mathrm{~K}$ reached 
Table 1 Lattice parameters of the synthesized powder before SPS and comparison of sintering temperatures and bulk density after SPS.

\begin{tabular}{|c|c|c|c|}
\hline \multirow{2}{*}{$\begin{array}{l}\text { Lattice parameter } \\
\text { before SPS [nm] }\end{array}$} & \multirow{2}{*}{$\begin{array}{c}\text { Sintering } \\
\text { temperature }[\mathrm{K}]\end{array}$} & \multicolumn{2}{|c|}{ Bulk density after SPS } \\
\hline & & $\left(\mathrm{g} / \mathrm{cm}^{-3}\right)$ & (\%T.D.) \\
\hline \multirow{6}{*}{0.3902} & 1513 & 5.03 & 96.27 \\
\hline & 1543 & 5.15 & 98.57 \\
\hline & 1573 & 5.14 & 98.49 \\
\hline & 1603 & 5.09 & 97.56 \\
\hline & 1633 & 5.16 & 98.91 \\
\hline & 1663 & 5.09 & 97.55 \\
\hline
\end{tabular}

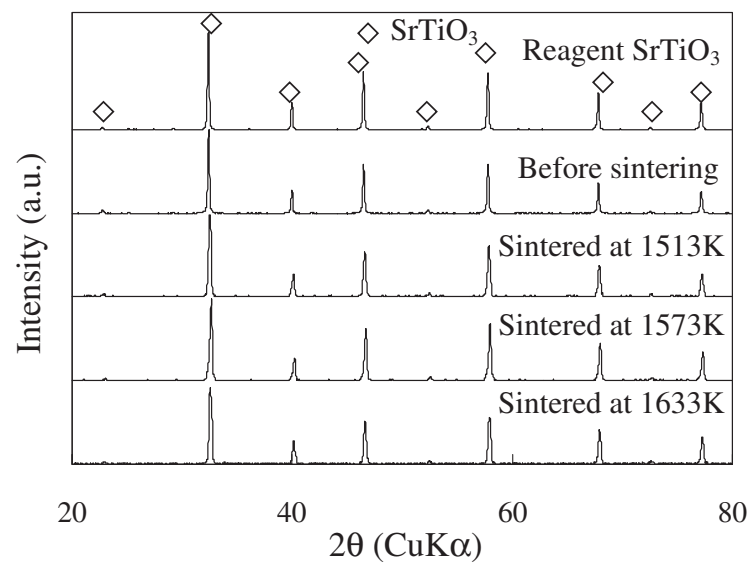

Fig. 2 X-ray diffraction patterns of SLTO before and after sintering at different temperatures, together with the data on reagent $\mathrm{SrTiO}_{3}$ for comparison.

$5.09 \mathrm{gcm}^{-3}$, which was $97.5 \%$ of their theoretical density (TD) $\left(\mathrm{TD}=5.24 \mathrm{gcm}^{-3}\right)$. In this study, the samples were sintered at different temperatures from 1513 to $1663 \mathrm{~K}$; however, bulk SLTO was not formed at a sintering temperature of $1663 \mathrm{~K}$, because of the formation of many intercrystalline cracks. Thus, sintering of SLTO was successfully carried out at temperatures from 1513 to $1633 \mathrm{~K}$.

Figure 2 shows X-ray diffraction patterns of SLTO before and after sintering at different temperatures, together with the data on reagent $\mathrm{SrTiO}_{3}$ for comparison. The XRD pattern of the analytical reagent $\mathrm{SrTiO}_{3}$ (99.9\% purity, Kojundo Chemical, Sakado, Japan) is also shown in this figure. The samples consist of cubic crystals. All peaks in this figure correspond very well to that of $\mathrm{SrTiO}_{3}$. This result indicates that SLTO with high purity can be prepared by CS. There is no difference between the XRD patterns obtained before and after SPS, indicating that no phase change takes place during sintering.

\subsection{Dependence of thermoelectric properties of CSed and SPSed SLTO on pressing direction during sintering}

SEM images of sections of the sample that are parallel and perpendicular to the pressing direction during sintering are shown in Figs. 3(a) and (b), respectively.

Figures 4(a) and (b) show the temperature dependence of parallel and perpendicular measurements on electric conductivity, $\sigma$, and Seebeck coefficient, $\alpha$, of CSed and SPSed SLTO, respectively. Parallel and perpendicular measure-
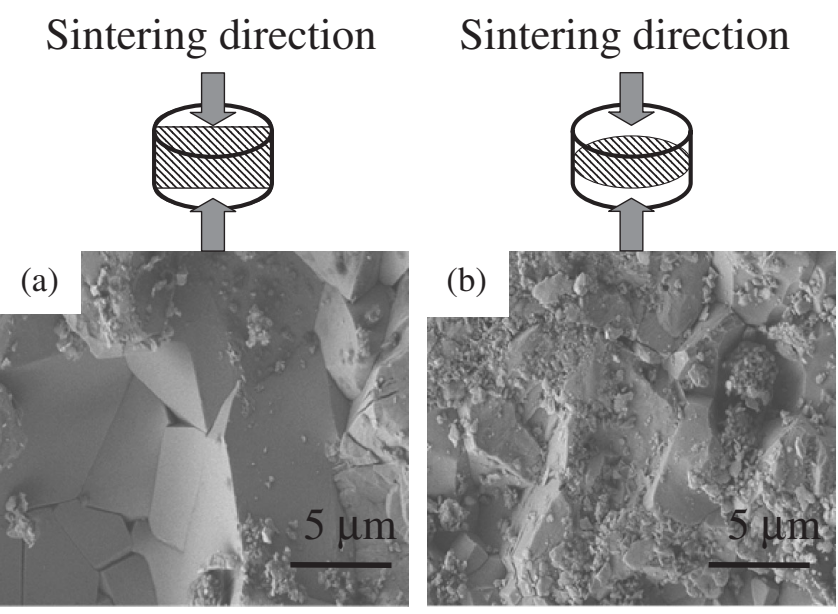

Fig. 3 SEM images of sections of the sample that are (a) parallel and (b) perpendicular to the pressing direction during sintering.

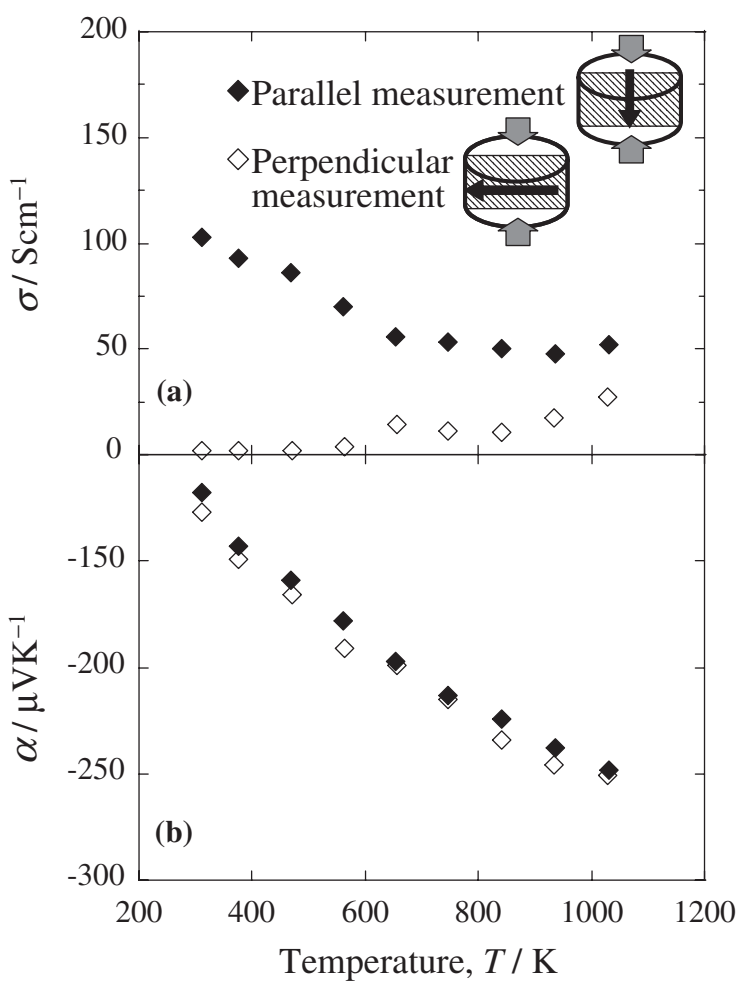

Fig. 4 Temperature dependence of parallel and perpendicular measurements on (a) electric conductivity and (b) Seebeck coefficient of combustion-synthesized and spark-plasma-sintered SLTO.

ments indicate that $\sigma$ and $\alpha$ were measured parallel and perpendicular to the pressing direction during sintering, respectively. As shown in Fig. 4(a), the curve of parallel measurement is above that of perpendicular measurement in the experimental temperature range. Additionally, from the SEM images shown in Fig. 3, it is found that there are many coarse grains in the parallel section of the samples (Fig. 3(a)), but there are only a few coarse grains in the perpendicular section of the samples (Fig. 3(b)). This implies that the powders attach easily along the pressing direction during sintering. Therefore, $\sigma$ from parallel measurement is larger than that from perpendicular measurement in the experi- 


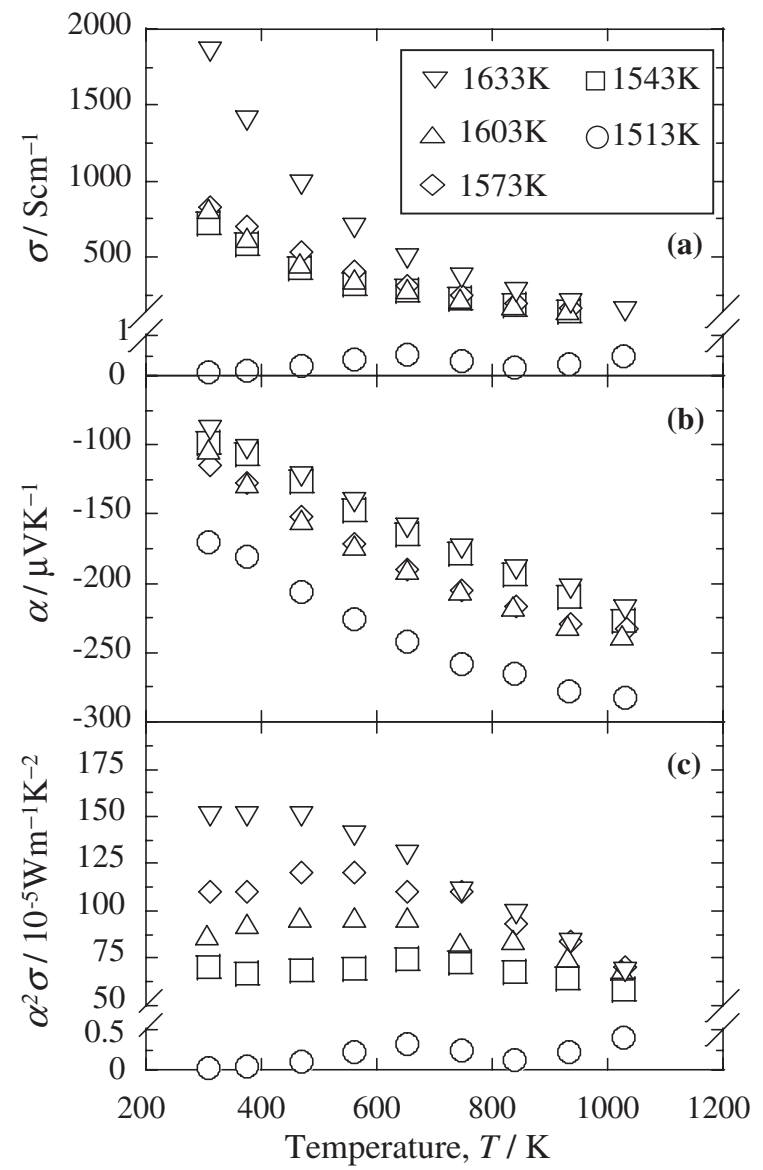

Fig. 5 Temperature dependence on (a) electric conductivity, (b) Seebeck coefficient and (c) power factor of combustion-synthesized and sparkplasma-sintered SLTO for various sintering temperatures.

mental temperature range. The values of $\alpha$, plotted in Fig. 4(b), are found to be almost equal, which indicates that the carrier densities of the two samples are equal.

\subsection{Temperature dependence of thermoelectric proper- ties of CSed and SPSed SLTO with various sintering temperatures}

Figures 5(a) and (b) show the temperature dependence on $\sigma$ and $\alpha$ of CSed SLTO for various sintering temperatures, respectively. With an increase in the temperature, the absolute values of $\alpha$ increase and $\sigma$ decreases, indicating a metallic behavior. In the experimental temperature range, with an increase in the sintering temperature, $\sigma$ increases and the absolute value of $\alpha$ decreases due to an increase in the carrier density. When a sample is sintered at a high temperature in a vacuum, a lot of oxygen inside crystal lattices are liberated and bulk SLTO are reduced. As a result, the sample changes into the nonstoichiometric compound $\mathrm{Sr}_{0.92} \mathrm{La}_{0.08} \mathrm{TiO}_{3-\delta}$, where $\delta$ is the oxygen defect content. Oxygen defects generate electrons as carriers. Thus, $\sigma$ increases and the absolute value of $\alpha$ decreases with an increase in the sintering temperature. The temperature dependence on power factor value of CSed SLTO for various sintering temperatures is shown in Fig. 5(c). As compared to the reference data, the power factor of $1.47 \times$ $10^{-3} \mathrm{Wm}^{-1} \mathrm{~K}^{-2}$ at room temperature is higher than that of

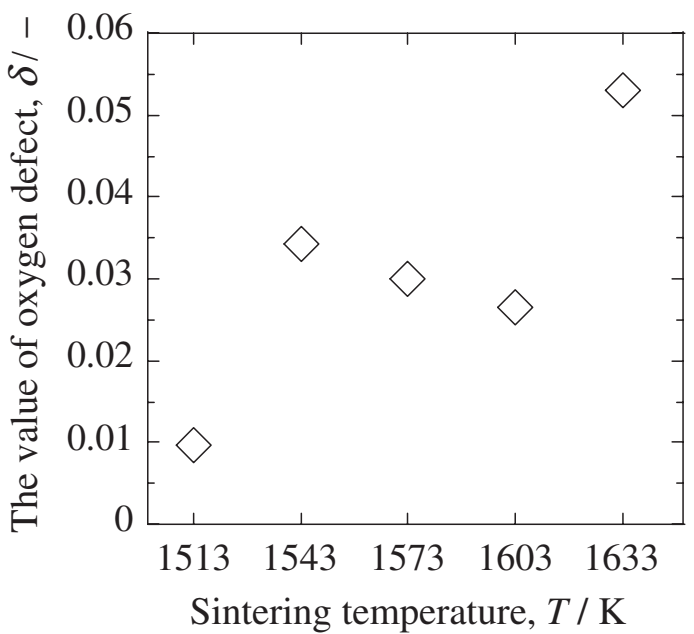

Fig. 6 The value of oxygen defect in the samples sintered at different sintering temperatures.

the single crystal samples reported by Ohta et al. ${ }^{8)}$ This result has proved that the combination of CS and SPS is a promising method to produce perovskite-oxide thermoelectric materials, and it is possible to enhance the thermoelectric properties of such materials by sintering them at high temperatures. The power factor is maximum at a sintering temperature of $1633 \mathrm{~K}$ in the experimental sintering temperature range, and it is minimum at a sintering temperature of $1513 \mathrm{~K}$. Among the samples, $\mathrm{Sr}_{0.92} \mathrm{La}_{0.08} \mathrm{TiO}_{3}$ sintered at $1633 \mathrm{~K}$ shows the largest power factor of $1.51 \times$ $10^{-3} \mathrm{Wm}^{-1} \mathrm{~K}^{-2}$ at $375 \mathrm{~K}$.

\subsection{Determination of $\delta$}

The value of $\delta$ was calculated under an assumption that the samples of $\mathrm{Sr}_{0.92} \mathrm{La}_{0.08} \mathrm{TiO}_{3-\delta}$ were oxidized to $\mathrm{Sr}_{0.92-}$ $\mathrm{La}_{0.08} \mathrm{TiO}_{3.00}$ using following equation:

$$
\delta=11.72 \Delta \mathrm{w} / \mathrm{w}_{\text {after }}
$$

In eq. (2), $\Delta \mathrm{w}$ and $\mathrm{w}_{\text {after }}$ denote the deference in weight before and after heating and weight after heating, respectively. The value of $\delta$ in the samples sintered at different temperatures is shown in Fig. 6. The largest value of $\delta$ is 0.053 in a sample sintered at $1633 \mathrm{~K}$, and the smallest value of $\delta$ is 0.0097 in a sample sintered at $1513 \mathrm{~K}$. The value of $\sigma$ of the samples sintered at different temperatures also shows a similar tendency, as shown in Fig. 5(a). As shown in Fig. 5(a), electric conductivities of specimens sintered at 1543,1573 , and $1603 \mathrm{~K}$ had almost equal values. Therefore, the $\delta$ values were also almost equal. Thus, an increase in the sintering temperature leads to an increase in $\delta$ content, thereby resulting in high $\sigma$.

\section{Conclusions}

We successfully synthesized La-doped $\mathrm{SrTiO}_{3}$ by the combination of CS and SPS and investigated the temperature and sintering temperature dependence of the thermoelectric properties of $\mathrm{Sr}_{0.92} \mathrm{La}_{0.08} \mathrm{TiO}_{3}$. Further, we investigated the effects of pressing direction during sintering on its properties and microstructure. The following results were obtained: 
(1) Combustion-synthesized $\mathrm{Sr}_{0.92} \mathrm{La}_{0.08} \mathrm{TiO}_{3}$ powders were successfully sintered by SPS at temperatures ranging from 1513 to $1633 \mathrm{~K}$.

(2) The average grain size of combustion-synthesized and spark-plasma-sintered SLTO increased with the sintering temperature. The maximum average grain size was $23.5 \mu \mathrm{m}$ when sintering at $1663 \mathrm{~K}$.

(3) Electric conductivity and microstructure of combustionsynthesized $\mathrm{Sr}_{0.92} \mathrm{La}_{0.08} \mathrm{TiO}_{3}$ were severely affected by the pressing direction during sintering. The combustion-synthesized powders were sintered well along the pressing direction during sintering; therefore, the power factor measured along pressing direction during sintering was more than twice that measured perpendicular to the pressing direction during sintering in the experimental temperature range.

(4) The power factor of combustion-synthesized and sparkplasma-sintered SLTO was maximum at the optimum sintering temperature of $1633 \mathrm{~K}$ because high-temperature sintering resulted in the introduction of oxygen defects and this oxygen defects produced electrons as carriers. Among the samples, $\mathrm{Sr}_{0.92} \mathrm{La}_{0.08} \mathrm{TiO}_{3}$ sintered at $1633 \mathrm{~K}$ showed the maximum power factor of $1.51 \times 10^{-3} \mathrm{Wm}^{-1} \mathrm{~K}^{-1}$ at $375 \mathrm{~K}$.

\section{REFERENCES}

1) L. Zhang, T. Tosyo, N. Okinaka and T. Akiyama: Mater. Trans. 48 (2007) 1079-1083

2) H. Muta, K. Kurosaki and S. Yamanaka: J. Alloy. Compd. 368 (2004) 22-24.

3) T. Maekawa, K. Kurosaki, H. Muta, M. Uno and S. Yamanaka: J.
Alloy. Compd. 387 (2005) 56-59.

4) H. Muta, K. Kurosaki and K. Yamanaka: J. Alloy. Compd. 392 (2005) 306-309.

5) T. Okuda, K. Nakanishi, S. Miyasaka and Y. Tokura: Phys. Rev. B. 63 (2001) 113104-1-113104-4.

6) H. Muta, K. Kurosaki and S. Yamanaka: J. Alloy. Compd. 350 (2003) 292-295.

7) H. Obara, A. Yamamoto, C. H. Lee, K. Kobayashi, A. Matsumoto and R. Funahashi: Jpn. J. Appl. Phys. 43 (2004) 540-542.

8) S. Ohta, T. Nomura, H. Ohta and K. Koumoto: J. Appl. Phys. 97 (2005) 034106-1-034106-4.

9) S. Ohta, T. Nomura, H. Ohta, M. Hirano, H. Hosono and K. Koumoto: J. Appl. Phys. Lett. 87 (2005) 092108-1-092108-3.

10) L. Zhang, T. Tosyo, N. Okinaka and T. Akiyama: Mater. Trans. 48 (2007) 2088-2093.

11) H. Ishikawa, K. Oohira, T. Nakajima and T. Akiyama: J. Alloy. Compd. 454 (2008) 384-388.

12) H. Simizu, M. Yoshinaka and K. Hirota: Mater. Res. Bull. 37 (2002) 1557-1563.

13) T. Nishimura, M. Mitomo, H. Hirotsuru and M. Kawahara: J. Mater. Sci. Lett. 14 (1995) 1046-1047.

14) I. J. Shon, Z. A. Munir, K. Yamazaki and K. Shoda: J. Am. Ceram. Soc. 79 (1996) 1875-1880.

15) J. J. Petrociv and R. E. Honnell: J. Mater. Sci. 25 (1990) 4453-4456.

16) Y. Liu, Y. Lin, Z. Shi, C.-W. Nan and Z. Shen: J. Am. Ceram. Soc. 88 (2005) 1337-1340.

17) Y.-h. Lin, Z. Shi, C.-W. Nan, Y. Liu and J. Li: J. Am. Ceram. Soc. 90 (2007) 132-136.

18) Q. Hu, P. Luo and Y. Yan: J. Alloy. Compd. 459 (2008) 163-168.

19) L. D. Zhao, B.-P. Zhang, J.-F. Li, H. L. Zhang and W. S. Liu: Solid State Sci. 10 (2008) 651-658.

20) X. Dong, F. Lti, L. Yang, Y. Zhang and X. Wang: Mater. Chem. Phys. 112 (2008) 596-602.

21) R.-C. Chang, S.-Y. Chu, Y.-P. Wong, C.-S. Hong and H.-H. Huang: J. Alloy. Compd. 456 (2008) 308-312. 